Cuadernos de Filología Clásica. Estudios Latinos

ISSN: 1131-9062

http://dx.doi.org/10.5209/CFCL.56186

\title{
Questiones de coitu. Estudio y edición ${ }^{1}$
}

Enrique Montero Cartelle ${ }^{2}$

Recibido: 15 de septiembre de 2016 / Aceptado: 25 de febrero de 2017

Resumen. En este trabajo se lleva a cabo la primera edición crítica de las anónimas Questiones de coitu del siglo XIII. Este texto se pone en su contexto escolástico, se estudian sus fuentes y se analiza su originalidad.

Palabras clave: Edición crítica; Questiones de coitu; medicina escolástica.

\section{[en] Quaestiones de coitu. Study and edition}

\begin{abstract}
This paper provides the first critical edition of the anonymous 13th century Questiones de coitu. This text is placed in its scholastic context, its sources are studied and its originality is analyzed. Keywords: Critical Edition; Questiones de coitu: Scholastic Medicine.
\end{abstract}

Sumario. 1. El marco literario. 2. Contenido y fuentes. 3. Tradición textual. 3.1 Manuscritos. 3.2. Manuscritos perdidos. 3.3. Relaciones entre los manuscritos. 3.4. El título. 3.5. El autor. 4. Edición crítica. 5. Referencias bibliográficas.

Cómo citar: Montero Cartelle, E., «Quaestiones de coitu. Estudio y edición», Cuad. filol. clás. Estud. lat. 37.1 (2017), 51-71.

\section{El marco literario}

1) Las Questiones de coitu se insertan dentro del género literario de las quaestiones carácterístico de la escolástica ${ }^{3}$. Este género alcanzó gran desarrollo en la filosofía y teología durante los siglos XIII y XIV en las principales universidades europeas, y se extendió también a la medicina.

Esta medicina escolástica participa de las mismas características que la filosofía y la teología del momento. De la misma forma que la filosofía incorpora y se basa en la asimilación del nuevo Aristóteles, la medicina escolástica se fundamenta en el

1 Este artículo se inscribe en el Proyecto «Estudios de medicina práctica en el Renacimiento. Las Centurias de Amato Lusitano» (FFI2013-4134-P).

1 Universidad de Valladolid (España).

E-mail: enrique@fyl.uva.es

3 Sobre el método escolástico en general, cf. Grabmann (1908-1911); Makdisi (1974); Kudlien (1974); AgrimiCrisciani 1988. 
nuevo Galeno, habitualmente conocido a través de los árabes (Montero 1999, 9-29; 2006, 173-207).

Entre otros rasgos, esta medicina tiene una acusada vertiente escolar (Paniagua 1966-67, 523 ss.; Agrimi-Crisciani 1988, 17 ss.). La enseñanza se basaba en la lectura de una autoridad, que venía acompañada de un comentario o glosa apropiados. Esta orientación no sólo predetermina muchas formas de exposición, sino también el tono teórico y doctrinal de estos tratados, así como la falta de orientación práctica. Las preguntas sobre este texto y ese comentario llegaron a alcanzar una forma altamente tecnificada, como las quaestiones ${ }^{4}$.

Las Questiones de coitu, que discuten cinco puntos problemáticos relativos al coito, entran perfectamente en este género ${ }^{5}$, como señala Laín Entralgo $(1978,235)$ :

La docencia en la facultad de medicina era fundamentalmente teórica. Fiel al esquema didáctico al uso (exposición de una tesis, explanación de ésta, objeciones, contra-objeciones, solución final) el maestro daba su lectio con el correspondiente texto a la vista, o resolvía los problemas que ocasionalmente se le presentaran (quaestiones quodlibetales).

Este género escolástico tiene antecedentes antiguos. Desde mucho tiempo antes existían quaestiones et responsiones de enorme simplicidad. Bien tengan estas cuestiones origen en las respuestas de los juristas romanos o bien haya sido Aristóteles quien organizó el sistema, como indicarían los "Problemas" pseudoaristotélicos (Lawn 1969, 21 y n. 9; Ventura 2004, 23-42), el caso es que fueron especialmente renovadas y enriquecidas por los maestros de la Escuela de Salerno. A partir de Salerno la fortuna de este género fue general en todas las universidades medievales. Las más conocidas en verdad son las Quaestiones salernitanae. Su carácter es eminentemente didáctico y consiste fundamentalmente en una pregunta en forma nítida y clara a la que se da una respuesta igualmente exacta de acuerdo con una teoría determinada. B. Lawn (1979) ha editado un corpus amplio en prosa anterior al año 1200 de origen salernitano, pero que también incluye cuestiones originadas en otras universidades como París o Montpellier. Estas cuestiones se interesaban por la filosofía natural y por la medicina, pero también incluían materias diferentes que manifestaban un interés enciclopédico.

Similar carácter tienen otras colecciones de la popular literatura de Problemata de la Edad Media (Ventura 2006, 120-121), entre las que destacamos los Problemata varia anatomica editados por Lind (1968), según un manuscrito de Bolonia fechado en 1408, pero que fue recopilado hacia finales del siglo XIII o comienzos del XIV, como fecha más temprana.

2) Como la medicina escolástica participa del interés por el conocimiento a través de las causas, que se adquiere por demostración, esto implica un alto grado de formalismo en la técnica demostrativa y la utilización de una lengua tecnificada en consonancia, como señala Norberg $(1969,89)$ : «le latin scolastique perdit le contact avec les belles-lettres et devint de plus en plus technique». Esta es la situación que reflejan

Un buen elenco de ellas las recoge N. G. Siraisi (1981) en un apéndice a su obra.

En Montero (2010, 87-94) se estudia este género literario en el contexto de los demás géneros literarios médicos medievales. 
las Questiones de coitu en sintaxis, lengua y estilo. La simplicidad y la monotonía son producto de la tecnificación de una lengua que en aras de la precisión elimina todo ornato. La fraseología argumentativa de la medicina está tomada de la filosofía y de la teología. Así, por ejemplo, en el planteamiento de la quaestio (iuxta hoc queritur utrum; tunc dubitatur quare), en los típicos argumentos, en las divisiones y subdivisiones temáticas (causa materialis, formalis, efficiens et finalis), en la articulación de la demostración por partes (de primo sic...) o su refutación (ad primum istorum dicendum; ideo non valet obieccio), en la conclusión lógica que no necesita ya explicitación (ergo et cetera; unde...), en las extensiones analógicas (similiter intelligendum est de...), etc.

\section{Contenido y fuentes}

Las Questiones de coitu se plantean los siguientes aspectos relativos a la actividad sexual:

\section{Primum est quid sit coitus.}

2. Secundum de causis eius.

3. Tercium de necessitate et iuvamento.

4. Quartum de diversitate seu diversificacione eius.

5. Quintum de differenti inmutacione qua corpus inmutatur ab ipso coitu.

Para ello, tal como se reconoce por las numerosas citas que lo atestiguan, se basan en una fuente fundamental: el Pantegni de 'Ali ibn Al-'Abbas traducido por Constantino el Africano en Montecassino a finales del siglo XI, apoyado por otras fuentes de modo muy secundario, de la forma siguiente, como indicamos en las notas al texto:

La cuestión primera se basa en Constantino, Pantegni, Theorica (1515) f. 25ra y b, V, cap. 107, apoyado por una definición de coito (Coitus est mutua accio maris et femine per instrumenta naturalia effecta ad speciem propagandam) que se repite literalmente en Problemata varia anatomica (Lind 1968) 55, 33-34, en la que se remite a Averroes (aunque en este autor no la hemos encontrado), lo que parece indicar que era una definición no original y conocida. También se menciona el capítulo De membris del libro I del Canon de Avicena (1527), que se encuentra en Canon 1.I.5.1, f. 85rb, 1. 9-23.

La cuestión segunda se basa en el mismo texto de Constantino, en ocasiones de modo literal, como lo referente a la causa materialis del coito e incluso la referencia a Hipócrates y Galeno de la causa finalis es una cita interna que aporta el mismo Constantino. El punto sobre la equivalencia en sangre del semen viene especificado también en Constantino, pero es tomado literalmente del libro III del Pseudo-Avicena De animalibus (=De natura animalium) (ca. 1500, f. 6v). Para la causa formalis utiliza la única mención que se encuentra en la Isagoge de Iohanitius (1513, f. 3ra.; Maurach 1978, $\mathrm{n}^{\circ}$ 40, 160) al coito 2,18.

Sin embargo, la especificación de los tres elementos necesarios para realizar el coito (calor, spiritus, humor), no se encuentra de manera específica en este texto de Constantino, pero sí en el De coitu 1 (Montero 1983), y en el Viaticum 6,1 (164ra18), cosa sorprendente, porque en estas cuestiones se advierte que el autor no conoce o 
no se sirve de estas obras de Constantino. No obstante, es una idea muy difundida en la Edad Media que aparece en muchos autores como Liber minor de coitu 2,3,2 (Montero 1987); Avicena, Canon 3.20.1,1-2, f. 279rab; B. de Gordon, Lilium (1542) 7.1.313v24; Tractatus de sterilitate (1542) 2.1.7; Gaddesden, Rosa (1492) 94ra51, etc. Ahora bien, la especificación sobre la patología que la falta de una estos elementos conlleva, es decir, la gomorrea, la satiriasis y el aproximeron, nos lleva directamente a afirmar que en este caso la fuente es el compendio de Plateario, Practica (Recio Muñoz 2016), caps. 54-56 que, como hemos estudiado en otro lugar (Montero-Herrero 2007, 315-337), es la fuente tanto de esta terminología como de la definición de los males que provoca para otros autores posteriores, como es el caso de Gilberto Ánglico (1510, f. 287) o del tratado más tardío del XIV sobre la esterilidad, el Tractatus de sterilitate (Montero 1993, caps. 1-3).

La cuestión tercera tiene la misma base con el apoyo de una cita literal de la Tegni de Galeno o, lo que es lo mismo, de la Ars parva, a la que sirve de introducción (Kühn I, 371).

La cuestión cuarta tiene las mismas características, aunque las ideas están dispersas en el Pantegni. Pero se esperaría una mención de la Ars parva de Galeno, que en esto es más preciso (Kühn 1, 371) o bien el De Veneriis (Kühn 5,911).

De la cuestión quinta tenemos que decir lo mismo. En Constantino, Pantegni, Theorica, V, cap. 107, se dedica la mayor extensión a la posición del coito con relación a la salud secundum res naturales, non naturales et contra naturam y así se recoge en estas Questiones con el apoyo de la misma cita de la Tegni de Galeno ya vista en la cuestión III. Esta relación entre la salud y la actividad sexual se centra dentro de la distinción tan usual y crucial en la medicina medieval árabe y luego latina, pero que tiene su origen en la medicina galénica, de las sex res non naturales. En efecto, Iohannitius en su Isagoge (García Ballester 1993, 105-115) fue el autor que organizó claramente las áreas relativas a la salud en res naturales (elementa, complexiones, humores, membra, virtutes, actiones, spiritus), res contra naturam (morbus, morbi causae y morbi accidentia:) y res non naturales (aer; motus et quies; cibus et potus; somnus et vigilia; inanitio et repletio (que limita a De coitu) y animae accidentia). Su difusión occidental se alcanzó a través de la traducción de Constantino el Africano del Pantegni de Haly Abbas, cuya terminología no difiere mucho de la de Iohannitius.

Las tres o cuatro (porque la primera en realidad son dos cuestiones) dudas planteadas tras esta exposición tienen en común que versan sobre el mismo texto de Constantino, del que se puede deducir la respuesta. Esa es precisamente la labor del autor de este opúsculo, para lo que echa mano del apoyo de otros textos como del mismo Pantegni, Theorica, VI, cap. 17; la Tegni de Galeno (Kühn I. 339; Articella f. 15r-15v.), del contraste tópico en la Edad Media sobre la existencia de un semen femenino (Gerlach 1937-1938, 177-193; Diepgen (1963); Martorelli (2000, 311-338.), aquí representado por la oposición entre Aristóteles y Avicena, a propósito de la cual se hace una típica conciliatio y, por último, una cita de adorno del Ars amatoria 1,332 de Ovidio, sobre la diferencia del grado de placer en el coito entre el hombre y la mujer.

De todo ello podemos deducir, en conclusión, que estas cuestiones, desde el punto de vista doctrinal, son una especie de paráfrasis del Pantegni, Theorica 5.107 de Constantino. Este texto, en efecto, proporciona al autor el entramado conceptual y las ideas básicas sobre las que responder a las cinco cuestiones, formuladas precisamente sobre el material que dicho capítulo le aporta, sin dejar ningún aspecto suyo fuera. 
Sobre esta base el autor se apoya en otras obras y auctoritates de renombre, algunas mencionadas ya en el propio Pantegni: En tres ocasiones se refiere a Avicena, dos de ellas al Canon y una al De animalibus. La Tegni de Galeno, a la que menciona en tres ocasiones, aunque en dos de ellas la idea sea la misma. Una sola vez cita la Isagoge de Iohannitius. En otra ocasión, por último, menciona a Aristóteles, en su De generatione animalium.

Ahora bien, todas estas citas son utilizadas como apoyo a las ideas expresadas en el Pantegni, por lo que las podemos considerar secundarias. Si tenemos en cuenta, por otro lado, que tanto la Isagoge de Iohannitius como la Tegni de Galeno son textos básicos que desde la Escuela de Salerno forman parte de la Articella $^{6}$, establecida a partir de las traducciones de Constantino, tenemos que la base de estas Questiones está en el corpus salernitano del Pantegni y la Articella, adornada con algunas citas de otros autores habituales y de gran renombre como el De generatione animalium de Aristóteles y el Canon de Avicena, que acabó desbancando al Pantegni de Contantino ya en el siglo XIII. En efecto, el Pantegni de 'Ali ibn Al-'Abbas (siglo X) fue traducido a finales del siglo XI por Constantino el Africano, y significó la obra de mayor influjo en la medicina medieval (Montero 1990, 65-88) hasta la traducción del Canon de Avicena (siglo X-XI), traducido en Toledo por Gerardo de Cremona en la segunda mitad del siglo XII, pero de difusión más tardía tras el segundo cuarto del siglo XIII y en el XIV (Siraisi ( 1987, 43-65).

Sobre esta base doctrinal formada por la paráfrasis de un capítulo del Pantegni, apoyada por las citas de las grandes autoridades, el autor de las Questiones montó toda una exposición dialéctica al modo de la escolástica, que es original suya. En ella destacan, además de la explicación en detalle de cada apartado, por un lado, la estructura general de la materia en cinco cuestiones claves que distribuyen de modo claro la problemática sobre el coito $\mathrm{y}$, por otro, la serie de cuestiones secundarias del apartado quinto de differenti inmutacione qua corpus inmutatur ab ipso coitu. En efecto, tras exponer los tres modos en los que el coito puede afectar al cuerpo, es decir, secundum res naturales, non naturales et contra naturam, siguiendo la exposición de Constantino, se plantea tres puntos dudosos primum est utrum coitus competat in regimine sanitatis. Secundum de debilitate que sequitur ad coitum. Tercium est de delectione que est in coitu, que van a dar pie a un desarrollo muy personal del autor, en el sentido de deducir, probar y demostrar con la lógica y la demostración característica de la escolástica, muchos y diversos problemas que se podrían plantear en contra de las ideas expresadas en la doctrina del Pantegni que acaba de exponer. Aquí es donde la literatura de Questiones y de Problemata adquiere toda su dimensión y justifica esta obra.

\section{Tradicion textual}

\subsection{Manuscritos}

El texto de las Questiones de coitu nos ha sido transmitido por los siguientes manuscritos:

6 Por esta razón añadimos la citas de la Isagoge y de la Tegni de la Articella, cuando las hemos localizado. 
P París, Bibliothèque Nationale, lat. 16195, fol. 23v-25r.

Su fecha es de finales del siglo XIII.

Referencias: Thorndike- Kibre1963, 386.

Según los datos que nos da el Catalogus codicum manuscriptorum Bibliothecae Regiae $(1744,45)$ estamos ante un códice misceláneo de medicina y filosofía natural de escasa extensión ( 37 folios en total), que contiene algunos pequeños textos latinos de medicina y filosofía natural en forma de tratado o de quaestiones, que son los siguientes: Epistola regis Egyptiorum Octaviano Augusto; De luminaribus; De febribus; De virtutibus; nuestro De coitu; De sensu communi; De anima; De motu cordis; De mixtione elementorum; De ocultis operationibus nature; De erroribus philosophorum et de unione anime ad corpus, finalizando con Divisio virtutum secundum philosophos. El contexto, en consecuencia, nos lleva de nuevo a la literatura de quaestiones.

K Kassel, LB u. Murhard. Bibl. d. Stadt. $4^{\circ}$ m. 8, f. 67r-68v.

Manuscrito escrito en torno al año 1418.

Por la descripción del Catálogo de Broszinski 1976, sabemos que es un manuscrito misceláneo de medicina y astrología, con algunas partes del pronóstico del tiempo. Dentro del campo médico, además de la obra que nos ocupa, tenemos obras como los Synomyma apothecariorum, el Carmen de urinis de Aegidius de Corbeil y varios tratados de Hipócrates como los Prognostica o los Aphorismi, además del De physicis ligaturis de Costa ben Lucca.

\section{U Uppsala, C 28, f. 25r-25v $+33 r$}

Estamos ante un manuscrito escrito en la primera mitad del siglo XIV.

De acuerdo con la descripción del Catálogo de Andersson-Schmitt \& Heldung 1988, este códice contiene obras de medicina, como los Prognostica o los Aphorismi de Hipócrates, la Isagoge de Iohannitius, el De viribus herbarum de Macer Floridus o el Liber lapidum de Marbodus Redonensis. Entre estas obras médicas se encuentran algunos tratados teológicos como el Compendium moralitatum de Iacobus de Lausanna o la Scala fidei de Albodrandinus de Tosca.

Con relación a nuestro tratado, lo más llamativo es que los ff. 25r-25v están censurados, mientras que el 33 r no lo está, probablemente porque no se apercibieron de que continuaba allí. Por ello, su testimonio es muy limitado.

El Explicit de este manuscrito que dice Explicit tractatus nobilis de cohitu quem scripsit Sweno dacus hace referencia al escritor danés de la primera mitad del siglo XII.

D Oxford, Bodleian Library, Digby, 150, fol. 101r-103r.

Manuscrito escrito a finales del siglo XIII.

Referencias: Thorndike-Kibre 1963, 386.

Este manuscrito contiene, según la descripción del catálogo de esta biblioteca, obra de Macray con revisiones y adiciones de Hunt-Watson 1999: Alberti Magni Summma philosophiae naturalis; Fragmentum ex abreviatione tractatus de sensu et sensatu; Disputacio Alberti super de morte et vita; Disputacio Alberti super de nutrimento et nutribili; Rogeri Bacon commentarius in libros Physicorum Aristotelis; nuestras Quaestiones de coitu; Quaestio de voluntate et intellectu; De phle- 
botomia; Extracciones de Thezauro pauperum; Commentarius in librum Aristotelis de bona fortuna per fratem Aegidium; Quaestiones sive Tractatus de intellectu potenciali editus a fratre Egidio; Quaestiones de anima; Commentarius (Aegidii de Columna) in tractatum Aristotelis de anima; Commentarius (Aegidii de Columna) in libros Metaphysicorum Aristotelis, concluyendo con una adición del siglo XIV que son unas Quaestiones breves de SS. Trinitate.

Todo lo cual nos muestra que nuestro tratado se encuentra dentro de la temática de filosofía natural y de medicina y que en cuanto a la forma, además de algunos comentarios y tratados breves, lo fundamental es la reunión de diferentes Quaestiones típicas medievales, denominando también con este nombre al tratado que aquí editamos.

\subsection{Manuscritos perdidos}

Laurent, en su obra Fabio Vigili et les bibliothèques de Bologne au début du XVI siécle d'aprés le ms. Barb. lat. 3185, (1943, 46), que, como dice el título, hace una recopilación de los manuscritos existentes en Bolonia en el siglo XVI, nos informa de que en el Convento de San Domenico existía con el n ${ }^{\circ}$ 194, un manuscrito cuyo título era: Senecae epistolae et quaedam alia opera eiusdem. La última de estas alia opera era un De coitu tractatus in quinque partes divisus, cuyo Incipit rezaba: De coitu quinque possunt notari. Primum quid sit coitus. Todos los datos apuntan a que se trataba del texto que editamos, hoy perdido.

\subsection{Relaciones entre los manuscritos}

Desde el punto de vista crítico, los testimonios manuscritos de este texto se engloban en dos ramas, derivadas de un modelo común, directa o indirectamente. Por un lado está la rama (a la que llamaremos y) conformada por los manuscritos $P, U$ y $K$, y, por otro, $D$. Los muy numerosos ejemplos del aparato crítico con esta relación muestran de modo fehaciente esta distribución.

La rama $y$ es la que tiene más solvencia crítica y la que aporta el texto más coherente, no ya por carecer de los errores evidentes que se advierten en $D$, sino por aportar la lectura correcta en casos tan complejos como es el caso del ap(p)roximeron frente a la lectura aptaximanton de $D$ en II,10. En todo caso, hay que advertir que el $U$ es un testimonio que sólo nos ayudará en una pequeña parte, dado que el manuscrito ha sufrido una censura brutal, al borrarse de modo muy efectivo con tinta todo el texto, que resulta ilegible. Sólo se salva de la censura el folio final, ya que se encuentra copiado, tras un salto, unos folios más adelante, cosa que no advirtió el censurador. Por lo demás, cada uno de estos manuscritos tiene personalidad propia, con lecturas peculiares que impiden hablar de una copia directa entre ellos; más bien pensamos en un modelo común con pasos intermedios. Esto es evidente en el tardío $K$, que tiene añadidos que evidentemente son de su cosecha.

El manuscrito $D$ es, en general, inferior críticamente. Da la impresión de ser un copista inexperto, porque, a pesar de tratarse de un texto pequeño, se confunde a menudo y tiene que rectificar de inmediato; además tiene faltas groseras de simple concordancia o de régimen de los casos tan evidentes que se advierte que no se trata de puros despistes; se come muchas veces el verbo est y, por último, también tiene saltos "de igual a igual". Pero todo ello no quiere decir que $D$ carezca de 
valor crítico, sobre todo después de la mitad del texto en adelante, donde sirve para confirmar algunas buenas lecturas.

Por todo ello, el texto se establece fundamentalmente por la concurrencia de $P$ y $K$ (y en su caso $U$ ), cuando hay diferencias críticas con $D$. No obstante, en los casos más conflictivos valoramos cada punto por crítica interna y/o externa.

Nuestro aparato crítico es completo y negativo. No recogemos, sin embargo, las cuestiones gráficas más banales como, por ejemplo, la variación ti/ci.

\subsection{El título}

El texto en los manuscritos aparece sin título. Sin embargo, conjeturamos que es Questiones de coitu con cierto grado de seguridad por diversas razones.

En primer lugar, el género literario de este texto es una quaestio típica medieval, de acuerdo con el sistema escolástico en el que se inserta, como hemos visto.

Este aspecto queda confirmado por el explicit de $D$ que reza Expliciunt questiones, lo que evidentemente se refiere a su forma literaria.

Este dato viene confirmado también por el contenido de los dos manuscritos que nos transmiten el texto, formados en su mayor parte por quaestiones, generalmente de filosofía natural. Como suele acontecer en obras de la misma clase, la especificación de que se trata de una quaestio no se repite al inicio de cada una de ellas.

En segundo lugar la explicitación de De coitu se debe a su temática, confirmada por el Explicit de U que reza: Explicit tractatus nobilis de cohitu quem scripsit Sweno dacus. Resulta además evidente del Incipit de los dos manuscritos De coitu quinque possunt notari, razón por la cual los dos catálogos de los manuscritos coinciden independientemente uno del otro en este título.

\section{5. Época}

El término ante quem es la época de dos manuscritos, el $P$ y el D, es decir, finales del siglo XIII.

El término post quem es la obra de Constantino (Pantegni yTegni), que se difundió a lo largo del siglo XII. Incluso, precisando un poco más, podemos ponerlo en la segunda mitad de este siglo, fecha de composición de la Practica de Plateario (Recio Muñoz 2016, 19-22).

No obstante, hay otros datos que nos permiten precisar más. En primer lugar la mención del Canon de Avicena, que fue traducido por Gerardo de Cremona en Toledo a mediados del siglo XII, pero cuya difusión general se alcanzó a lo largo del siglo XIII e incluso el XIV, como hemos señalado.

En segundo lugar, el encuadre de este texto dentro de la medicina escolástica. Además, los datos que hemos visto en B. Lawn (1993, 67-84) nos indican que las quaestiones escolásticas más elaboradas, como las que nos ocupan no tienen entidad en el campo de la medicina hasta después de mediados del siglo XIII.

Por último, la coincidencia de la definición inicial de coito con los Problemata varia anatomica nos plantea un problema. Si se hubiese tomado de aquí, nos situaríamos a finales del XIII o comienzos del XIV, pero estimamos que ambos la toman de una fuente común desconocida para nosotros, aunque se remita a Averroes.

En consecuencia, debemos concluir que esta obra es de la segunda mitad del siglo XIII, poco anterior, por lo tanto, a los manuscritos que nos la transmiten. 


\subsection{Autor}

Como hemos señalado, sorprende mucho que el autor de este texto se base sólo en el Pantegni de Constantino y no conozca otro de los textos traducidos por Constantino sobre esta temática, el Liber de coitu, en el que se profundiza mucho en esta cuestión y cuenta con un aparato de fuentes relativamente importante. Además su difusión fue grande, en particular a partir de los grandes enciclopedistas del siglo XII. De hecho es una fuente básica para estas cuestiones en los Problemata varia anatomica. El autor, si bien menciona la Tegni de Galeno, que conoce seguramente por la Articella, tampoco muestra conocer otras obras básicas sobre el tema de Hipócrates y Galeno. También sorprende que no haya entrado a fondo en Avicena, en particular el libro III, Fen XX del Canon de Avicena, que tiene probablemente la mayor y más completa exposición sobre la vida sexual desde el punto de vista médico del momento.

Por otro lado es también muy significativo que este texto no aporte nada doctrinalmente sobre la teoría del Pantegni, limitándose a formular al modo escolástico las ideas del Pantegni y a darle forma de quaestio, con la adición de alguna que otra auctoritas, como Aristóteles o Avicena.

Esta situación nos lleva a pensar que se trata de un ejercicio escolar universitario de medicina o bien de un estudioso de la filosofía natural, que ha dado forma de quaestio a las ideas de un capítulo del Pantegni sobre cinco puntos de interés relativos al coito, sin complicar su tarea con todas las fuentes disponibles.

De hecho este tipo de preguntas tiene paralelos claros en las típicas Quaestiones medievales que circulaban ampliamente. Hay cuestiones similares en las colecciones salernitanas ya indicadas del siglo XIIl editadas por B. Lawn. De la misma forma se dan quaestiones paralelas en la literatura de "Problemas" como la mencionada de L. R. Lind. Todo lo cual permite establecer un lazo entre la literatura de problemas que remonta a Aristóteles y las genuinas quaestiones medievales. En todo caso estamos ante un producto escolar medieval.

Como señalamos, no merece crédito alguno la noticia aislada del Explicit de este manuscrito relativo a la autoría del tratado Explicit tractatus nobilis de cohitu quem scripsit Sweno dacus, que hace referencia a este escritor danés de la primera mitad del siglo XII, anterior incluso a la difusión de algunas de las fuentes mencionadas en las Questiones. Se utiliza este nombre de la zona donde se copia el tratado simplemente para darle mayor autoridad. 


\section{Edición crítica}

\section{$<$ QUESTIONES DE COITU $>$}

De coitu quinque possunt notari. Primum est quid sit coitus. Secundum de causis eius. Tercium de necessitate et iuvamento. Quartum de diversitate seu diversificacione eius. Quintum de differenti inmutacione qua corpus inmutatur ab ipso coitu.

I. De primo sic. Coitus est mutua accio maris et femine per instrumenta naturalia effecta ad speciem propagandam ${ }^{7}$. Satis manifestatur hec diffinicio, sed cum dicit ad speciem propagandam, quia hec debet esse intencio forme operi imponentis, sed pauci homines reperiuntur, ut dicit Constantinus in V sue Theorice, capitulo "De coitu": Omnes homines fere coitum appetunt propter delectacionem, pauci vero spe gignendorum filiorum ${ }^{8}$. Ab Avicenna potest extrahi talis diffinicio, primo Canonis, capitulo "De membris": Coitus est operacio animalibus attributa ad servandam generacionem per membra conveniencia, que essenciam animalitatis non sunt introeuncia, sed necessitatem et iuvamentum habent; necessitatem ut servent speciem, iuvamentum ut complementum forme detur et complexio vel composicio masculina et femenina servetur? .

Tit. coni. ex inc. et expl.

Pr. 1 est: om. $K \cdot 2$ ante iuvamento scr. et del. innato $D \cdot \mathbf{3}$ eius: temporis coitus $K \cdot \mathbf{4}$ post differenti add. et $K$. I, $\mathbf{6}$ effecta: effectum $D \cdot 7$ esse: om. $D \cdot \mathbf{8}$ imponentis: impones $P \|$ ante omnes add. quia $D \|$ coitum: iter. $D \cdot \mathbf{1 1}$ : animalibus: animalis $D \|$ servandam: servandum $D \cdot \mathbf{1 2}$ essenciam: materia $D \|$ introeuntia: intro $K \cdot \mathbf{1 3}$ necessitatem: necessitate $D \cdot \mathbf{1 4}$ composicio: composita $D$.

$7 \quad$ Aparece esta definición en Problemata varia anatomica (Lind 1968, pp.55, 33-34): Queritur quid sit coitus? Respondetur secundum Averroim quia est mutua actio maris et femine per instrumenta a natura ad esse specierum conservandum producta. Según ello, la fuente procede de Averroes, pero no la hemos localizado ni en el Colliget, ni en los comentarios a Aristóteles. Probablemente, como señala el editor en sus notas, muchas de estas citas no corresponden a la realidad, como sería este caso.

8 Constantino, Pantegni, Theorica, V, cap. 107, f. 225a: Omnes fere homines delectationem tantum in coitu appetunt, pauci vero gignendorum spe filiorum.

9 Aquí se hace un arreglo inicial de Avic. Canon 1.1.5.1, f. 85rb, 1. 9-23. Cuando este habla de los membra vero principalia sunt membra que primarum virtutum que in corpore existunt, que ad hoc sunt necessarie ut singulare aut species perdurent como son el corazón, el cerebro y el hígado, se añade aliud quartum quod speciei existit principium, et sunt testiculi qui uni rei sunt necessarii et ad rem aliam iuvativi, cuya explicación toma: Necessitas vero est ad sperma generandum quod genealogiam conservat. Iuvamentum vero est ad hoc ut complementum forme detur et complexio masculina et feminina conservetur, que speciebus animalis accidentia sunt inseparabilia et nec sunt ex rebus in animalitatis essentiam ingredientibus. 
II. De secundo debemus notare quod tria requiruntur ad coitum, calor vivificans et dissolens, spiritus sive ventositates qui humorem dissolutum impellat, et humor qui dissolvatur ${ }^{10}$. Si autem aliquid istorum desinerit, causa est diversarum egritudinum in membris genitalibus. Verbi gracia, si humor habundet et deficiat spiritus, fit quedam passio que gomorrea apellatur, scilicet, involuntaria spermatis emissio. Si spiritus et ventositas habundet et humor deficiat, fit alia passio que satiriasis appellatur et est inmoderata virge ereccio. Si utrumque deficiat, fit alia passio que dicitur aproximeron, id est, inoperacio parcium genitalium ${ }^{11}$.

Item fiunt alie cause, sicut materialis, formalis, efficiens et finalis:

Causa materialis est sperma, cuius natura, sicut dicit Constantinus in V sue Teorice, auctoritate Galeni, est aerea ignea, que de claro et mundo sanguine nascitur, membra principalia nutritura. Unde cum homo nimium coit, desiccat corpus eius, eo quod materia nutrimenti aufertur membris ${ }^{12}$; unde, sicut dicit Avicenna in 4 De naturis animalium: Cum homo nimium coit, magis debilitatur quam si quadragies tantus exiret per flebothomiam, quia sperma est de nutrimento parato ad membra nutrienda ${ }^{13}$.

Causa efficiens duplex est: prima est mediata, ut est apprehensio rei delectabilis, et secunda est inmediata, quam comitatur maxima delectacio, quam siquidem delectacionem natura ingenita est hac de causa, cum enim turpe sit officium ab animalibus refutare et ideo natura tantam delectacionem posuit in coitu, ne ipso abhominato animal deperiret ${ }^{14}$.

Causa formalis est concussio quam sequitur caliditas, ut dicit Iohannicius ${ }^{15}$.

\begin{abstract}
II, 16 tit. De causis coitus marg. add. $D \|$ quod: quoniam $D \|$ ante coitum add. scilicet $D \cdot \mathbf{1 7}$ ante spiritus add. et $K \cdot \mathbf{1 9}$ habundet: habundat $P \cdot \mathbf{2 0}$ deficiat: deficiunt $D \|$ ante appellatur scr. et del. dicitur $D \|$ gomorrea appellatur: vocatur gomorrea $K \cdot \mathbf{2 1}$ scilicet. om. $D \|$ spermatis emissio: $\operatorname{tr} . K \|$ habundet: habundat $P \cdot \mathbf{2 2}$ satiriasis appellatur: vocatur satiriasis $D \|$ et: ut $P \|$ ante virge $a d d$. in $P \cdot \mathbf{2 3}$ aproximeron $P$ approximeron $K$ aptaximanton (?) $D \cdot \mathbf{2 5}$ sicut: sicud scr. semper $K \|$ efficiens, formalis: tr. $D \cdot 27$ ignea corr. ex Pantegni, Theorica, V, cap. 107, f. 25a: natura inquit spermatis aeree et ignee est nature: igne $K D$ materia $P \cdot \mathbf{2 8}$ nascitur: nascuntur $D \|$ nutritura: nutritivo $D \cdot \mathbf{3 1}$ quadragesies tantus corr. ex Avic. De animalibus $6 f$. $6 v$ : quadragies exiret de suo corpore sanguis tantus: quadragesies tantum $P K D \|$ flebothomiam: flebotomiam $D \cdot \mathbf{3 3}$ duplex est: $t$ r. $K \|$ est: et $P \cdot \mathbf{3 5}$ hac de causa: de hac causa $D$ - 36 turpe: tempore $D \|$ officium: effectum D $\|$ refutare: refutareur $D \cdot \mathbf{3 8}$ post caliditas add. in toto $K$.
\end{abstract}

10 Cf. Constantino, De coitu 1 y Viaticum 6,1 (164ra18). Como hemos indicado, es una idea muy difundida en la Edad Media que aparece en muchos autores como Liber minor de coitu 2,3,2; Avic. Canon 3,20.1.1-2, f. 279rab; Gordon, Lilium 7.1,313.v24; Tractatus de sterilitate 2.1.7; Gaddesden, Rosa 94ra51, etc.

11 La fuente es Plateario, Practica, cap. 54-56 ( $c f$. Recio Muñoz, 2016, 706-721), en el que se inspiraron, a su vez, otros autores, como Gilberto Ánglico f. 287 o Tractatus de sterilitate II, caps 1-3.

12 Pantegni, Theorica, V, cap. 107, f. 25a: Unde Galenus dixit in libro custodiende sanitatis: natura inquit spermatis aeree et ignee est nature; eius ergo complexio calida est et humida necessario, quia de puro et clarifico sanguine generatur, unde fundamenta principalium membrorum nutriuntur.

13 Avicenna, De animalibus, 6 f. 6v: Et quando de homine exit sperma multum discoloratum et debilitatur suum corpus magis quam si quadragies exiret de suo corpore sanguis tantus, quia sperma est res habilitata ad transeundum in membra et cum exit, aufertur naturale nutrimentum membri. La idea también está en Pantegni, Theorica, V, cap. 107, f. 25a.

14 Es la idea con la que comienza Constantino su exposición del Pantegni, Theorica, V, cap. 107. Se encuentra muy resaltada en Const. De coitu, pr., e imitada por todos los que después trataron el tema. Se encuentra ya en Galeno, De usu partium 14.2 (Kühn 4, 143-144).

15 En la exposición de las seis cosas no naturales referidas a la salud Iohannitius en su In Artem Parvam Galeni Isagoge, Articella, Venetiis 1513 f. 3ra (G. Maurach, nº 40, 160), dice del coito: coitus haec praestat corpori: desiccat corpus et minuit naturalem virtutem, ideoque infrigidat, multotiens vero ex multa concussione corpus calefacit. 
Causa finalis diversificatur secundum diversos opifices. Secundum naturales causa est finalis ut conservetur species, ut quod homo in se non poterat servare in alio servaretur. Secundum medicos causa finalis coitus est conservacio sanitatis, quia secundum Galenum et Ypocratem coitus custodiende sanitatis est fomes, quia per ipsum expelluntur superfluitates, que, si in corpore remanerent, passiones egritudines generarent ${ }^{16}$.

III. De tercio notandum quod eius utilitas est quadruplex ${ }^{17}$.

Prima humani generis reparacio et conservacio et hoc secundum legem debet esse precipua.

Secunda est caloris naturalis et spirituum confortacio eo quod superfluitates que per coitum expelluntur, si putrefient, possunt calorem naturalem corrumpere.

Tercia est corporis alleviacio. In coitu enim, cum corpus calefiat et superfluitates expellantur, corpus alleviatur.

Quarta est malarum cogitacionum sollicitudinum et suspicionum exclusio, quod contingit propter maximam delectacionem. Sed quod diximus de coitu intelligimus quod fit tot et tantis intervallis, ut dicit Galenus in Tegni, quod corpus nec dissolucionem nec laborem senciat, sed utens eo se ipso factus levior videatur ${ }^{18}$.

39 finalis est: $t$ r. $P \|$ post est $a d d$. coitus $P \cdot \mathbf{4 1 - 4 2}$ : coitus est $K$ est coitus $P$ est $D \cdot \mathbf{4 3}$ : est: om. $K \cdot \mathbf{4 4}$ ante generarent add. generant seu $D$.

III, 45 tit. De utilitate $a d d . K \|$ quod: quoniam $D \cdot \mathbf{4 6}$ ante humani $s c r$. et del. uma $D \cdot 47$ esse: om. $D \cdot 48$ naturalis calor: tr. $D \cdot \mathbf{4 9}$ coitum: totum corpus $K \|$ putrefient: putrefierent $D \cdot \mathbf{5 1}$ alleviacio: levigacio $K \|$ calefiat: caleficiat $D \cdot \mathbf{5 2}$ expellantur: expelluntur $D \cdot \mathbf{5 4}$ propter maximam: maxime propter $D \|$ post diximus add. hic $D \cdot \mathbf{5 5}$ ante intelligimus $a d d$. quod $P \|$ fit: sit $D \cdot \mathbf{5 6}$ nec $^{1}$ : non $K$.

16 Esta es la idea con la que comienza el Pantegni, Theorica, V, cap. 107, donde está también la mención de Hipócrates y Galeno. Esta misma idea reaparece en Const. De coitu, pr. y se encuentra muy resaltada en Const. Pantegni, Theorica, V, cap. 107, f. 25a: Hipp. et Ga. dixerunt: Coitus sanitatis custodiende fomes est unus, alli contra... especificando a continuación las mismas, entre otras razones.

17 Estas razones se encuentran todas ellas dispersas en Pantegni, Theorica, V, cap. 107 y se hacen tópicas en la Edad Media. Cf. Const. De coitu, 9-10 y Avic. Canon 3.20.1, cap. 1 ss.

18 Véase el texto de la Tegni recogido en Iohannitius, In Artem Parvam Galeni Isagoge (Articella, Venetiis $1513 \mathrm{f}$. 29vb-30ra: Venereorum vero secundum Epicurum nullus usus salubris sed verum ex intervallis tantis ut in usibus coitus neque dissolutionem sentiat et levior ipse se ipso videatur factus et melius dormiens (que corresponde a Galeno, Ars med. 24, Kühn I, 371). 
IV. De quarto notandum quod coitus diversificatur sic, quia quidam est moderatus, quidam inmoderatus. Moderatus est qui fit, ut dicendum est, et huius est facere que dicta sunt, scilicet, calorem confortare, corpus alleviare, malam suspicionem removere, et iste est qui fit semel vel bis in septimana. Inmoderatus est qui fit qualibet hora, aliquando ter vel quater vel plures de nocte secundum potenciam vel impotenciam coeuntis et iste infrigidat et desiccat, quia membris aufert nutimentum.

Item quidam est parvus, quidam magnus. Magnus in quo est multa delectacio et multa spermatis emissio, parvus qui opposito ( $s c$. modo) $\mathrm{fit}^{19}$.

V. De quinto intelligendum est quoniam diversificatur accio vel inmutacio quam facit coitus in corpore tribus modis, scilicet, secundum res naturales, non naturales et contra naturam ${ }^{20}$.

Secundum res naturales, ut secundum complexionem, quidam sunt frigidi, quidam calidi et sic de aliis, et eciam de complexionibus compositis. Qui ergo sunt calidi et humidi de complexione pingues et carnosi et rufi in colore potentes fiunt ad coitum; qui vero sunt frigide et sicce complexionis minus appetunt et minus possunt, et si frequenter coeant, defeccionem incurrunt. Alii sunt frigidi et humidi et hii parum appetunt, cum tamen in opere multum possunt. Alii sunt calidi et sicci et hii multum appetunt et parum possunt.

IV, 58 tit. De diversitate coitus add. $K D \cdot \mathbf{5 9}$ dicendum: dictum $K \cdot \mathbf{6 5}$ post Magnus add. est $D \cdot \mathbf{6 5 - 6 6}$ multa delectacio: magna del. $K$ maxima del $D \cdot \mathbf{6 6}$ ante emissio add. spermatis $P \|$ opposito (sc. modo) fit: opposito modo habet fieri $K$, qui ex V, 137-139 add.: Sed iuxta hoc queritur secundo utrum coitus temperatus competat in regimine sanitatis, et videtur quod non, quia nichil quod calorem naturalem diminuit compatitur in regimine sanitatis et coitus temperatus calorem naturalem diminuit, ergo et cetera. Probacio huius: in coitu temperato et in quibus coitu diminuitur · V, 67 marg. De mutacione corporis a coitu $a d d$. $D \cdot \mathbf{7 0}$ non natur. et contra nat.: et contra nat. et non natur. tr. $D \|$ ante quidam add. quia $P \cdot \mathbf{7 2}$ colorore scr. et corr. colore $D \cdot \mathbf{7 3}$ frigide et sicce: $\operatorname{tr} D \cdot \mathbf{7 4}$ coeant: coeunt $P \cdot \mathbf{7 5}$ cum: et $D \|$ post opere $a d d$. sunt $K \cdot \mathbf{7 6}$ Alii ... parum possunt: om. $P$.

19 Estas ideas se encuentran dispersas en Pantegni, Theorica, V, cap. 107 y coinciden con las de Galeno, Ars med. 24 (Kühn I, 371; De Veneriis (Kühn V 911).

20 La larga exposición general sobre el efecto en la salud secundum res naturales, non naturales et contra naturam procede en su conjunto de Pantegni, Theorica, V, cap. 107. Estas ideas también se hacen tópicas a partir de Constantino De coitu, 5 y 8 así como de Avic. Canon 3.20.1.1-2,f. 279rab. La consecuencia final que concluye con la cita de la Tegni aparece en el tratamiento de la complexión de los testículos en Articella f. 15r-15v (Galeno, Ars med. 13, Kühn I, 339). 
Secundum res non naturales eciam diversificatur coitus secundum cibum et potum, quia, si fiat corpore existente plectorico sive repleto, preparat ad egritudines malas, ut ad arteticam et ad molliciem nervorum et in corpore humores viscosi gravantur. Si vero fiat corpore existente inanito, corpus extenuatur vehementer, unde aliquando spasmus et defeccio sequitur; tamen si contingat peccare, sicut dicit Galenus, melius est facere corpore pleno quam inanito, calefacto quam infrigidato. Similiter intelligendum est de concurrentibus anime, quia non debet fieri post maximum gaudium vel dolorem. Similiter intelligendum est de temporibus anni, quia in estate non debet fieri nec autumpno, eo quod illa tempora sunt inconvenientia, sed in illis temporibus debet fieri in quibus magis habundat materia spermatis, scilicet, humiditas, et ideo magis competit in vere, quia tunc habundat humiditas, et tunc magna fit conmocio humorum. Postea magis competit in hieme, quia tunc maxime habundat humiditas.

Item secundum res contra naturam diversificatur coitus accio, quia sunt quidam melancolici, quidam hereosi ${ }^{21}$, alii malorum humorum habundantiam habentes, qui secundum differentes passiones istas utuntur coitu et secundum hoc diversas acciones efficit in corporibus eorum; in melancolicis melancoliam aufert, in hereosis malam cogitacionem, in aliis nimiam plenitudinem. Diversificatur eciam secundum diversitatem accidencium superveniencium accioni coitus. Quidam enim coeuntes abhominacionem paciuntur, in ore siccitatem et oculorum concavitatem, ut sunt colerici, qui, si abstineant multum nocet eis, quia inmediate replentur vasa eorum propter assiduitatem desiderii in qua sunt.

Consequenter notandum quibus magis competit coitus et videtur quod calidis et humidis et eciam carnosis, quia dicit Galenus in Tegni quod calidi et sicci magis appetunt et magis leduntur. Calidi et humidi magis appetunt et minus leduntur, et repletis similiter competit propter evacuacionem, frigidis et extenuatis et convalescentibus ab egritudine nocet, et debilibus et pueris eciam nocet, quia ab ipsis evacuatur quod deberet in membrorum substanciam convenienti. Decrepitis nocet propter emissionem spermatis et caloris naturalis qui pauci sunt in ipsis.

\footnotetext{
77 eciam ante res non naturales tr. $K \cdot \mathbf{7 8}$ post potum add. et accidentia eciam tempora anni secundum cibum et potum $K \|$ plectorico: plectoricum $D \cdot \mathbf{7 8 - 7 9} \mathrm{ad} . .$. ad... ad: om. $D \cdot \mathbf{8 0 - 8 1} \mathrm{Si}$... extenuatur: $\operatorname{marg}$. $s c r . D \cdot \mathbf{8 1}$ defeccio: defectus $D \|$ post si add. sicud dicit Galenus $K \cdot \mathbf{8 3}$ inanito: inani $D \cdot \mathbf{8 7}$ quibus: quo $D \cdot \mathbf{8 9}$ magna: maxima $D \cdot \mathbf{9 2}$ hereosi: heretici $K \cdot \mathbf{9 5}$ malam cogitacionem: malas cogitaciones $D \cdot \mathbf{9 7}$ enim: eciam $D \cdot \mathbf{9 9}$ multum: nimium $D \cdot \mathbf{1 0 1}$ marg. Cui magis convenit coitus add. $D \|$ competit: convenit $D \cdot \mathbf{1 0 3 - 1 0 4}$ calidi --- leduntur: om. $P \cdot \mathbf{1 0 4}$ repletis: replecionem $P \|$ ante evacuacionem scr. et del. ea $D \cdot \mathbf{1 0 5}$ post debilibus add. quia (et $D$ ) ab ipsis (ipso $P$ ) evacuatur quod deberet in membrorum substanciam (subst. mem. tr. $P$ ) consequenti, decrepitis eciam nocet (ex $l$. $105-106$ iter.) $P D \|$ post pueris add. parvis $D \cdot \mathbf{1 0 6}$ eciam: enim $D \cdot \mathbf{1 0 6 - 1 0 7}$ in membrorum substanciam convenienti $P$ : in subst. conv. memb. $K$ in subst. memb. conv. $D$.
}

21 Sobre el morbus hereos o “mal de amor”, Cf. Ciavolella (1976); Wack (1990); Peri (1996). Para el término, cf. DILAGE s.v. 
Circa partem istam tria vel 4 possunt principaliter dubitari: primum est utrum coitus competat in regimine sanitatis. Secundum de debilitate que sequitur ad coitum. Tercium est de delectatione que est in coitu.

De primo sic, et videtur quod coitus competat in regimine sanitatis quia, quod calorem naturalem confortat et animam letificat et ingenium acuit et iram sedat, et cetera, competit in regimine sanitatis. Coitus est huiusmodi secundum quod dicit Constantinus in V sue Theorice, capitulo "De coitu"22, ergo et cetera. Oppositum videtur quia nihil quod corpus infrigidat et desiccat competit in regimine sanitatis; coitus est huiusmodi secundum quod dicit auctor iste, ergo et cetera. Si ergo dicatur quod, cum dicit auctor iste quod coitus infrigidat et desiccat, intelligit de coitu superfluo.

Tunc dubitatur quare non tangit effectus coitus temperati, quoniam videtur quod deberet facere, quia in parte precedenti non solum tangit effectus exercicii superflui, sed eciam effectus exercicii moderati. Similiter determinat effectus sompni moderati et superflui, quare videtur quod similiter in parte ista deberet tangere effectus coitus temperati et non solum superflui.

Ad primum istorum dicendum quod coitus temperatus et temporibus determinatis competit in regimine sanitatis.

Ad aliud quod opponitur dicendum quod auctor iste est breviloquus et introductorius et ideo solum tangit effectus qui frequenter accidunt et iste est superfluus. Unde notandum quod sunt aliqui coeuntes causa generacionis et isti sunt pauci; sunt alii coeuntes causa sanitatis conservande et isti adhuc pauci sunt. Sunt alii coeuntes causa delectacionis et isti sunt multi, et isti superflue coeunt et ideo quia effectus coitus superflui frequencius pluribus accidit, effectus autem coitus moderati parum et paucis accidit. Ideo auctor iste solum tangit effectus coitus superflui et non temperati, sed sic non est de exercicio et sompno. Effectus enim exercicii temperati et sompni temperati frequenter et multis accidit.

\footnotetext{
-109 marg. Questiones de coitu add. D || Circa --- dubitari: Questiones solent fieri contra premissa $K \|$ Circa partem istam: om. $K \|$ tria vel 4 possunt: possunt tria vel 4 tr. $D \cdot \mathbf{1 1 3}$ naturalem confortat: $t r . K \cdot 114$ ante coitus $a d d$. et $K$. $115 \mathrm{~V}$ : causa D $\cdot \mathbf{1 1 6}$ ante videtur $s c r$. et del. arguitur $D \|$ corpus: om. $D \cdot \mathbf{1 1 7}$ ante coitus $a d d$. sed $K \cdot \mathbf{1 1 8} \operatorname{ergo} \ldots$ iste: om. $D \|$ auctor iste: Avicenna et Iohannicius et philosophus in libro de morte et vita $K \cdot \mathbf{1 1 9}$ desiccat: sicat $K \|$ post desiccat add. corpus $D \cdot \mathbf{1 2 3}$ in parte ista: om. $K$ deberet: debeat $K \cdot \mathbf{1 2 4}$ solum superflui: $t r . D \cdot \mathbf{1 2 5}$ et temporibus: in temp. $K \cdot \mathbf{1 2 7}$ iste: ille $K \cdot \mathbf{1 2 8}$ effectus: om. $K \cdot \mathbf{1 2 8 - 1 2 9}$ accidunt et iste est superfluus: accidit et isti sunt superflui $K \cdot 130$ sanitatis: delectacionis $K \|$ adhuc: om. $K \cdot \mathbf{1 3 1}$ delectacionis: sanitatis conservandi sunt $K \|$ post isti add. pauci valde sed qui coeunt causa delectacionis sunt multi $K \cdot \mathbf{1 3 2}$ et: om. $P \cdot \mathbf{1 3 3}$ coitus moderati: $\operatorname{tr} . D \|$ et: vel $P \|$ accidit: accidunt $P \cdot \mathbf{1 3 3 - 3 4}$ auctor iste: $\mathrm{om} . K \cdot \mathbf{1 3 4}$ solum tangit: $t r . K \|$ coitus: om. $K \cdot \mathbf{1 3 5}$ temperati: moderati $K$ || ante temperati scr. et del. moderati $D \|$ ante sompni scr. et del. $\operatorname{sp} D \cdot \mathbf{1 3 6}$ accidit : accidunt $P$.
}

22 Pantegni, Theorica, V, cap. 107, f. 25 b. 
Sed iuxta hoc queritur secundo utrum coitus temperatus competat in regimine sanitatis et videtur quod non, quia nihil quod calorem naturalem minuit competit in regimine sanitatis. Coitus temperatus calorem diminuit, ergo et cetera. Probacio huius: in coitu temperato et in quolibet temperato et in quolibet coitu diminuitur aliquid de calore naturali et spiritibus quare coitus calorem naturalem diminuit. Oppositum videtur auctoritate omnium auctorum. Ad hoc dicendum quod aliquid emittitur de calore naturali et spiritibus. Dicendum quod si emittatur aliquid, quia tamen tunc evacuatur materia que calorem naturalem habebat obtendere et diminuere. Ideo plus postea fortificatur calor quam debilitatur in ipsa emissione spermatis ${ }^{23}$.

Tercio dubitatur qui magis debilitantur sive ledantur in coitu, an viri an mulieres et videtur quod mulieres quia quod est debilius plus leditur in qualibet operacione, ergo mulieres plus leduntur quam viri ${ }^{24}$. Item plus leduntur mulieres in accepcione cibi superflui quam viri, ergo et in coitu superfluo. Oppositum patet, quia dicit Gelenus in Tegni ${ }^{25}$ quod complexio sicca plus leditur et debilitatur in coitu quam humida, sed viri sunt sicci respectu mulierum, ergo viri plus leduntur in coitu quam mulieres. Item sperma in viris magis est digestum et in mulieribus magis aquosum, sed maior est debilitacio sive lesio in emissione digesti et puri quam indigesti et impuri, ergo et cetera. Item in coitu viri sunt agentes et mulieres pacientes sed agens magis gravatur quam paciens, ergo et cetera. Ad hoc dicendum quod viri magis debilitantur sive leduntur in coitu quam mulieres et causa huius est quia plus et purius et cum maiori pena mittunt et plus de calore naturali et spiritibus; fit enim in viris maxima concussio. Ad obiecta dicendum quod si eodem modo operantur ad coitum, sequeretur quia plus lederetur mulier quam vir, sed non est sic, ut visum est in solucione, quia viri plus molestantur in coitu propter maximam concussionem factam a viris et non a mulieribus et ideo non valet obieccio. Ad aliud dicendum est quod non est silere de cibo et coitu, quia mulieres sunt debilioris caliditatis quam viri respectu virorum, plus leduntur in accepcione cibi superflui quam viri, qui sunt caloris forcioris; non sic autem est in coitu, quia in eo non ita laborant sicut viri.

\footnotetext{
- 137 marg. Questio de coitu temperato add. $D \|$ secundo: om. $K \cdot \mathbf{1 3 9}$ minuit: diminuit $D \cdot \mathbf{1 4 1}$ spiritibus: spermatis $D \cdot$ 141-142 quare ... diminuit: et cetera $D$. $\|$ spiritibus ... diminuit: om. $D \|$ spiritibus: spermatibus $D \cdot \mathbf{1 4 2 - 1 4 3}$ auctoritate ... auctorum $P$ : auctori et omnibus auctoribus $D$ a multis auctoribus $K \cdot \mathbf{1 4 2}$ post dicendum add. breviter $D$ quod coitus temperatus ad hoc quod opponitur $K \cdot \mathbf{1 4 4}$ si: et si $D \|$ emittatur aliquid: aliquis emittatur de calori naturali $K \cdot 145$ materia: aliquid de materia $K \|$ habebat ... diminuere: heberit obtundit et diminuit $P \|$ obtendere: obtundere $K \|$ Ideo: et ideo $P \cdot \mathbf{1 4 6}$ calor: om. $D \cdot \mathbf{1 4 7}$ marg. Quis magis appettant add. $\cdot \mathbf{1 4 8 - 1 4 9}$ et videtur ... ergo: quia mulieres sunt debiliores respectu virorum, sed quod debilius est magis leditur in quolibet operacione, ergo $D$. 149 ergo mulieres plus leduntur (in coitu $a d d . K$ ) quam viri: sed mulieres sunt debiliores viris, ergo et cetera $P \cdot \mathbf{1 4 9}$ $\mathbf{1 5 0}$ plus leduntur ... mulieres: et cetera $D \cdot \mathbf{1 5 3}$ in viris: viri $D \cdot \mathbf{1 5 4}$ magis est: $t r . D \cdot \mathbf{1 5 6}$ agens magis: $t r . K \cdot \mathbf{1 5 8}$ causa: racio $P \cdot 159$ spiritibus: spermatibus $D \cdot 160$ maxima concussio: labore maximo $K \|$ Ad obiecta dicendum: om. $K \|$ post si add. vir et mulier $D$ post lederetur scr. et del. vir $D \cdot 162$ maximam: magnam $P$. $\cdot 163$ a: om. $P \cdot 164$ est: om. $K U \|$ coitu: cohitu $s c r$ semper $U \cdot \mathbf{1 6 4 - 1 6 5}$ sunt ... caliditatis: que debilioris caliditatis sunt $D \cdot \mathbf{1 6 5}$ caliditatis: caloris vel caliditatis $U \|$ respectu virorum: om. $U \cdot 166$ sic autem: tr. $U \cdot 167$ post viri add. quia et cetera $a d d$. $U$ •
}

\footnotetext{
3 Este punto está también en Pantegni, Theorica, V, cap. 107, f. 25b sin mencionarlo.

24 Es la idea general de Pantegni, Theorica, V, cap. 107, f. 25 b.

25 La mención de la Tegni es la ya antes indicada de Articella f.15r-15v. (Galeno, Ars med. 13, Kühn I, 339).
} 
Quarto queritur qui magis delectentur in coitu an viri vel mulieres, et videtur quod mulieres quia illud dicit Ovidius: Acrior est nostra plusque furoris habet ${ }^{26}$. Item dicit Constantinus in VI sue Theorice, capitulo "De doloribus et deliciis" 27 , et hoc probat quia mulieres delectantur dupliciter et in recepcione spermatis et in emissione, sed viri in emissione tantum, ergo plus delectantur mulieres quam viri. Contra sperma in viris plus est digestum quam in mulieribus, ergo plus expulsioni adaptatum, ergo plus est sollicita natura in eius emissione quam in emissione spermatis mulierum et propter hoc accidit maior delectacio, ergo et cetera. Ad hoc dicendum quod viri plus delectantur in coitu quam mulieres et causa huius est quia in viris sperma magis est digestum et ita plus calidum et humidum quam sperma mulierum, unde ibi plus est coniunccio convenientis cum convenienti, quando transit sperma viri per virgam et per loca nervosa et ita fit ibi quasi quedam concordancia et armonia, que sunt frigida et sicca; non autem sic fit de spermate mulieris et ideo maior fit delectacio in viris quam in mulieribus. Unde dicendum est quod viri magis delectantur qualitative, sed mulieres plus delectantur quantitative. Per hoc patet solucio ad obiectum, quia dicit Constantinus quod mulieres plus delectantur, hoc est, in pluribus vicibus, quia in recepcione et in emissione, sed non intensius, sed pocius viri, ergo et cetera.

Iuxta hoc queritur utrum delectacio sit in coitu et videtur quod non, ad minus in coitu superfluo, quia in coitu superfluo fit separacio convenientis a convenienti et ex hoc causatur dolor et non delectacio, quia videtur quod in coitu superfluo non sit delectacio, sed dolor. Ad hoc dicendum quod in coitu superfluo est delectacio. Ad illud quod opponitur quod, licet in decisione non sit delectacio, tamen postquam descendit ad membra inferiora, in ipsa emissione est delectacio. Iuxta hoc queritur utrum mulieres spermatizant et videtur quod non auctoritate philosophi in libro De animalibus, ubi monet istam questionem in XVII, qui dicit quod mulieres non spermatizant ${ }^{28}$. Oppositum videtur auctoritate Avicenne in primo capitulo ${ }^{29}$, ubi dicit quod creati sumus ex duplici spermate, scilicet, viri et femine, ergo mulieres spermatizant, quod

\footnotetext{
168 tit. Qui magis delectantur in cohitu viri vel mulieres $a d d$. $U \|$ vel: an $P \cdot 169$ quia ... Ovidius: secundum illud Ovidii $K \cdot 171$ post Item add. hoc $D \|$ quia: quod $D \cdot 172$ post ergo add. quoque $U \cdot 173$ in viris: viri $D \|$ plus est: tr. $D .174$ est sollicita: solicita est $\operatorname{tr} D \cdot \mathbf{1 7 7}$ causa huius: $\operatorname{tr} K \|$ magis est: $\operatorname{tr} U \cdot \mathbf{1 7 9}$ plus est: $\operatorname{tr} U \cdot \mathbf{1 8 1}$ Que ... sicca: om. $K \cdot \mathbf{1 8 2}$ est: om. $K U \cdot \mathbf{1 8 3}$ qualitative: qualitatem $D \|$ quantitative: quantitatem $D \cdot \mathbf{1 8 4}$ solucio: $\operatorname{sic} D \cdot \mathbf{1 8 6} \operatorname{ergo:}$ ideo $P K \cdot \mathbf{1 8 7}$ tit. Questio erit utrum delectacio sit in cohitu $a d d$. U marg. De delectacione coitus $D \cdot \mathbf{1 8 9}$ a convenienti: om. $P \|$ et: sed $D$ - 191 illud quod: om. $U \cdot \cdot 193$ ante Luxta hoc marg. add. Utrum mulieres spermatizent aut non $U \cdot 195$ post XVII add. animalium $K \|$ qui: ubi $D \cdot 196$ capitulo: Canonis $K \|$ mulieres: femine $U \|$ quod: om. $D$
}

26 Ovidio, Ars am. 1,342: Omnia feminea sunt ista libidine mota / acrior est nostra plusque furoris habet.

27 Este punto se basa en la exposición de Pantegni, Theorica, VI, cap. 17, f. 28rb, titulado De letitiis sive doloribus y que concluye: Delectatio in coitu maior est in mulieribus quam in masculis, quia masculi delectantur tantum in expulsione superfluitatis. Mulieres dupliciter delectantur, et in suo spermate expellendo et masculi recipiendo ex vulve ardentis desiderio, texto clave para todo el pensamiento medieval al respecto. Sobre ello véase Montero (2008), 97-116, donde se indican otros textos paralelos.

28 La negación de Aristóteles, De gener. animalium I.19.727b, de la existencia del semen femenino frente a los médicos en general, seguidores de Hipócrates y Galeno, representados aquí por Avic. Canon 3.20.1,3, f. 279rb279va, por ej., es una polémica habitual en la Edad Media. Cf. Bologne (1998, 46 ss.); S. Laurent (1989, 63 ss.), etc. Para las conciliaciones de ambas teorías contrapuestas, tal como hace el autor de las Quaestiones, cf. Martorelli (2000, 311-338).

29 Avic. Canon 3.20.1,3, f. 279rb-279va. 
concedendum est. Ad auctoritatem philosophi dicendum quod, cum dicit spermatizat intelligit extra sive in alio sicut viri, vel intelligit quod non spermatizant sperma digestum respectu spermatis virorum. Iuxta hoc queritur quare viri coeunt ita quolibet tempore, non sic autem alia animalia ${ }^{30}$. Ad hoc dicendum quod hoc est racione temperacionis complexionis necnon nutrimenti magis convenientis, similiter et porci, quia post hominem sunt temperacioris complexionis, similiter et alii. Item queritur quare mulieres magis appetunt coitum post impregnacionem quam femine aliorum animalium. Ad hoc dicendum quod hoc contingit quia in mulieribus non solum est appetitus naturalis, sed et animalis et ideo possunt habere imaginacionem coitus precedentis sive delectacionis que fuit in coitu precedenti et ideo coitum appetunt, sed sic non est imaginacio in feminis aliorum animalium et ideo post impregnacionem non appetunt coitum, sicut mulieres.

\footnotetext{
- 198 post dicendum add. est $D \|$ post dicit add. quod non $U$ non $K \cdot \mathbf{2 0 0}$ ante Luxta hoc marg. add. Quare viri non coheunt in quolibet tempore et non cuncta animalia $U \cdot \cdot 203$ magis: om. $K \|$ convenientis: conveniencia $D \|$ sunt: om. $D \|$ temperatioris: temperacionis $D \cdot \mathbf{2 0 4}$ Quare mulieres magis appetunt cohitum quam femine aliorum animalium post impregnacionem marg. add. $U$.

In fine add. Expliciunt questiones $D$ Explicit tractatus nobilis de cohitu quem scripsit Sweno dacus $U$.
}

30 La idea de que el ser humano ha superado el ciclo reproductivo en la cuestión sexual es habitual en la literatura de problemas. Cf. Lawn (1969, B23; Ba97, etc.), o bien Lind $(1968,57)$. 


\section{Referencias bibliográficas}

Agrimi J. - Crisciani CH. (1988), Edocere medicos. Medicina escolastica nei sc. XIII-XIV, Nápoles, Guerini e Associati.

Articella, per Petrum Bergomensem, Venetiis 1513.

Andersson-Schmitt M. \& Heldung M. (1988), Mittelalterliche Handschriften der Universitätsbibliothek Uppsala. Katalog über die C-Sammlung, Bd. 1: Handschriften C I-IV, 1-50, Stocolmo, Almqvist \& Wiksell.

Avicena, (1527), Avicenne Liber canonis medicinae, cum castigationibus Andree Bellunensis... translatus a magistro Gerardo Cremonensi..., Venetiis, In aedibus Luce Antonii Junta florentini.

Avicenna, De animalibus per magistrum Michaelem Scotum de Arabico in Latinum translatus (ca. 1500) s. n., Venetiis.

Bazan C. - Frazen G. - Wippel J.W. - Jacquart D. (1985), Les questions disputées et les questions quodlibétiques dans les facultés de Théologie, de Droit et de Médecine, Turnhout, Brepols.

Bologne, J.C. (1998), La naissance interdite, Stérilité, avortement, contraception au Moyen Age, París, Orban.

Broszinski, H. (1976), Die Handschriften der Murhardschen Bibliothek der Stadt Kassel und Landesbibliothek Bd. 3,1: Manuscripta Medica, Wiesbaden, Harrassowitz.

Bultot R. et alii, Les genres littéraires dans les sources théologiques et philosophiques médiévales. Definition, critique et explotation, Actes du Colloque Intern. de Louvain-la-Neuve, Université Catholique du Louvain, Lovaina La Nueva 1982.

Cadden, J. (1993), Meaning of Sex Difference in the Middle Ages. Medicine, Science and Culture, Cambridge University Press, Cambridge.

Catalogus codicum manuscriptorum Bibliothecae Regiae (1974), vol. IV, Parisiis, e typographia regia.

Ciavolella M. (1976), La “malattia d'amore” dall'Antichità al Medioevo, Roma, Bulzoni.

Conde, P.P. - Montero Cartelle, E. - Herrero Ingelmo M. C. (eds.), (1999), Tractatus de conceptu. Tractatus de sterilitate mulierum, Universidad de Valladolid, Valladolid.

Constantino el africano (1983), Constantini Liber de coitu. El tratado de andrología de Constantino el Africano, E. Montero (ed.), Universidad de Santiago de Compostela, Santiago de Compostela 1983.

Diepgen, P. (1937), Die Frauenheilkunde der alten Welt, Handbuch der Gynäkologie, Munich, Bergman.

Diepgen, P. (1963), Frau und Frauenheilkunde in der Kultur des Mittelalters, G. Thieme Verlag, Stuttgart.

A.A.V.V., (2017), Diccionario Latino de Andrología, Ginecología y Embriología desde la Antigüedad al siglo XVI (DILAGE), Barcelona, FIDEM.

Gaddesden, Juan, (1942), Rosa Anglica medicinae seu Practica, Papiae, Joannes Antonius Biretta.

García Ballester L. (1993), «On the Origin of the 'Six non-natural Things' in Galen», J. Kallesh-D. Nickel (edd.), Galen und das Hellenistische Erbe, Beihefte zu Sudhoffs Archiv, Stuttgart, 105-115.

Gerlach, W. (1937-1938), «Das Problem des 'weiblichen Samens' in der antiken und mittelalterlichen Medizin», Sudhoffs Archiv 30, 177-193.

Gilbertus Anglicus (1510) = Compendium medicine Gilberti Anglici tam morborum universalium quam particularium nondum medicis sed et cyrurgicis utilissimum, per Iacobum Saccon, expensis Vicentii de Portonariis, Lugduni 1510. 
Glorieux, P. (1925-1935), La littérature quodlibetique de 1269 à 1320, 2 vols., Kain 1925 y J. Vrin, París, Le Saulchoir.

Gordon, Bernardo de (1542), Bernardi Gordonii omnium aegritudinum a vertice ad calcem opus... quod Lilium Medicinae appellatur, Parisiis, Dionysius Ianotius.

Grabmann, M. (1908-1911), Die Geschichte der Scholastischen Methode, Freiburg-im-Breisgau, Herder 1908-1911 (reimpr. Graz, Akademische Druck 1957. Hay traducción italiana de Florencia, La Nuova Italia 1980).

Hunt, R.W. - Watson, A.G. (1999), Bodleian Library Quarto Catalogues IX, Digby Manuscripts, Oxford, Bodleian Library.

Kudlien, F. (1974), «Dialektik und Medizin in der Antike», MHL 9,187-200

Kühn, C.G. (ed.) (1821-1833), Galeni Opera omnia, Leipzig, Cnobloch (reimpr. Hildesheim, Olms, 1964-1965).

Laín Entralgo, P. (1978), Historia de la Medicina, Barcelona, Salvat.

Laurent M.-H. (1943), Fabio Vigili et les bibliothèques de Bologne au début du XVI siécle d'aprés le ms. Barb. lat. 3185, Città del Vaticano, Biblioteca Apostolica Vaticana, (reimpr. Modena 1987).

Laurent, S. (1989), Naître au Moyen Âge. De la conception à la naissance: la grossesse et l'accouchement (XII-XV siècle), Paris, Le Léopard d'Or.

Lawn, B. (1979), The Prose Salernitan Questions, Oxford University Press, Oxford.

Lawn, B. (1969), I quesiti salernitani, Di Mauro, Nápoles (trad. ampliada de The Salernitan Questions, Oxford University Press, Oxford, 1963).

Lawn, B. (1993), The Rise and Decline of the Scholastic "Quaestio Disputata” with Special reference on its Use in the Teaching of Medicine and Science, Leiden, Brill.

Lind L.R. (1968), Problemata varia anatomica: the University of Bologna, MS. 1165, ed., University of Kansas, Lawrence.

Littré, É. (1839-1863), Oeuvres complètes d'Hippocrate, vol. I-X, París, J.-B. Baillière.

Makdisi G. (1974), «The Scholastic Method in Medieval Education. An Inquiry into its Origins in Law and Theology», Speculum 49, 640-661.

Martorelli, R. (2000), «La medicina scolastica tra galenismo e aristotelismo», Studi medievali 41-1, 311-338.

Maurach, G. (1978), «Johannitius. Isagoge ad Techne Galieni», Sudhoffs Archiv 62.3, 148174.

Montero Cartelle, E. (1987), Liber minor De Coitu. Anónimo salernitano, Estudio y Ed. crítica, Universidad de Valladolid, Valladolid.

Montero Cartelle, E. (1990), «Encuentro de culturas en Salerno: Constantino el Africano, traductor», en Rencontres de cultures dans la philosophie médiévale. Traductions et Traducteurs de l'antiquité tardive au XIVe siècle, Hamesse, J.-Fattori, M., (eds.), Lovaina la Nueva-Cassino, Brepols, 65-88.

Montero Cartelle, E. (1993), Tractatus de sterilitate. Anónimo de Montpellier, Universidad de Valladolid, Valladolid.

Montero Cartelle, E. (2006), «La recepción de los textos médicos en la Edad Media: de Salerno a Toledo», en Actas del XI Congreso Español de Estudios Clásicos, vol. 3, Sociedad Española de Estudios Clásicos, Madrid, 173-207.

Montero Cartelle, E. (2008), «El mito de Tiresias: medicina, erotismo y literatura», en La transmisión de la Ciencia desde la Antigüedad al Renacimiento, Santamaría Ma T. (ed.), Ediciones de la Universidad de Castilla-La mancha, Cuenca, 97-116.

Montero Cartelle, E. (2010), Tipología de la literatura médica latina: Antigüedad, Edad Media, Renacimiento. FIDEM, Textes et études du Moyen Âge, 53, Porto, Brépols. 
Montero Cartelle, E. - Herrero Ingelmo, Mª C. (1998-1999), «Costantino Africano e il recupero dei testi greci antichi di medicina», Schola Salernitana. Annali III-IV, 9-29.

Montero Cartelle, E. - Herrero Ingelmo, Ma C. (2007), «Las deformaciones léxicas en los textos salernitanos: aproximeron y gomorrea», La scuola medica salernitana: gli autori e i testi, Convegno internazinale. Universitá degli Studi di Salerno, 3-5 novembre 2004, Firenze, SISMEL. Ed. del Galluzzo 2007, 315-337.

Norberg, D. (1969), Manuel pratique du latin médiéval, Picard, París.

Ottosson P.-G. (1984), Scholastic Medicine and Philosophy. A Study of Commentaries in Galen's Tegni, ca. 1300-1450, Nápoles, Bibliopolis.

Paniagua J. A, (1966-67), «Arnaldo de Vilanova médico escolástico», A.I.H.M. 18-19, 517532.

Peri M. (1996), Malato d'amore. La medicina dei poeti e la poesía dei medici, Catanzaro, Rubbettino.

Ramírez de Veger, A. - Socas, Fr. (1995), P. Ovidio. Obra amatoria II: Arte de amar, Madrid, CSIC.

Recio Muñoz, V. (2016), La Practica de Plateario. Edición crítica, traducción y estudio, Firenze, Sismel, Edizioni del Galluzzo.

Siraisi, N.G. (1981), Taddeo Alderotti and his Pupils, New Jersey, Princeton University Press.

Siraisi, N.G. (1987), Avicenna in Renaissance Italy. The Canon and Medical Teaching in Italian Universities after 1500, New Jersey, Princeton University Press:

Thorndike, L. - Kibre, P. (1963), A Catalogue of Incipits of Mediaeval Scientific Writings in Latin, Cambridge, Mass. 1963.

Ventura I. (2004), «Questions and Encyclopaedias: Some Aspects of the Late Medieval Reception of the Pseudo-Aristotelian Problemata in Encyclopaedic and Scientific Nature», en A.A. MacDonald-M.W. Twomey (eds.), Schooling and Society. The Ordering and Reordering of Knowledge in the Western Middle Ages, Peeters, Lovaina-París-Dudley, 23-42.

Ventura I. (2006), «Aristoteles fuit causa efficiens huius libri: On the Reception of Pseudo-Aristoteles Problemata in Late Medieval Encyclopedic Culture», en P. De Leemans-M. Goyens (eds.), Aristotle's Problemata in Different Times and Tongues, Lovaina, Leuven University Press.

Omnia opera Ysaac (1515), vol. I-II, Lugduni, in officina probi viri Iohannis de Platea.

Wack, F. Mary (1990), Lovesickness in the Middle Ages. The Viaticum and its Commentaries, Philadelphia, University of Penssylvania Press. 